Volume 8. No. 8, August 2020

International Journal of Emerging Trends in Engineering Research

Available Online at http://www.warse.org/IJETER/static/pdf/file/ijeter63882020.pdf https://doi.org/10.30534/ijeter/2020/63882020

\title{
Fuzzy Based Classification of X-Ray Images with Convolution Neural Network
}

\author{
Simhadri Chinna Gopi ${ }^{1}$, Kaveti Kiran Kumar ${ }^{2}$, Mothukuri Veerabrahmam ${ }^{3,}$ Yalanati Ayyappa $^{4}$ \\ ${ }^{1,2,3}$ Assistant Professor, CSE Department, Vignan's Foundation For Science Technology and Research University, \\ Vadlamudi, Andhra Pradesh, India. \\ ${ }^{4}$ Department of Computer Science and Engineering, Koneru Lakshmaiah Education Foundation, Vaddeswaram, \\ AP, India.
}

\begin{abstract}
Nowadays technology is changing from day to day in almost all fields, that too much in the medical field. There are some techniques that are still continuing for a long time due to their popularity and their robustness. One among them is X-ray which is used for detecting the fractures in a bone. In some rural areas where the medical facility is poor it very difficult to have sufficient orthopaedics for treatment. Hence a computerized effective and robust X-Ray image classification technique is proposed which is the initial step for fracture detection. In this work a combination of high boost filtering technique, Fuzzy C means clustering, Statistical feature extraction technique along with different kinds of classifiers like Support Vector Machine (SVM), Convolution neural network, and Back-Propagation neural network (BPNN). A detailed comparison is done with the accuracy rates with all classifiers where the Convolution neural network gives an accuracy rate of $94.2 \%$ when compared to other neural networks. Hence Convolution neural network $(\mathrm{CNN})$ is considered
\end{abstract}

Key words: X-Ray, CNN (Convolution neural network), Support Vector Machine (SVM), Backpropagation neural network(BPNN)

\section{INTRODUCTION}

Medical imaging is growing day-to-day life. Advancement in medical field leads to the penetration into the body and further used for detecting abnormalities in the body. Among the available MRI, CT, X-Ray techniques re popular in use. Among the available options X-Rays are mostly used due to its low cost compare to CT Scan and MRI Scan. X-Rays images are very popular in medical field in case of accidents or any traumas in a human body. It is necessary to automate the classification and further detection of fractures as it helps in taking immediate decisions for the orthopaedicians. For detecting the fractures in a bone it is very necessary to classify what kind of X-Ray image it is .in case of Computer Aided Detection (CAD) of fractures. This is the reason it has become the initial step as classification of X-ray image. For classification of X-Ray images a dataset of totally 2000 X-Ray images are collected from the NRI Medical Hospital, Department of Orthopaedics, Guntur, where 400 images are of skull,400 images are of Leg,400 images are of hand,400 images are of chest, and remaining 400 are neck. Detailed architechture of medical imaging is presented in the figure.1.

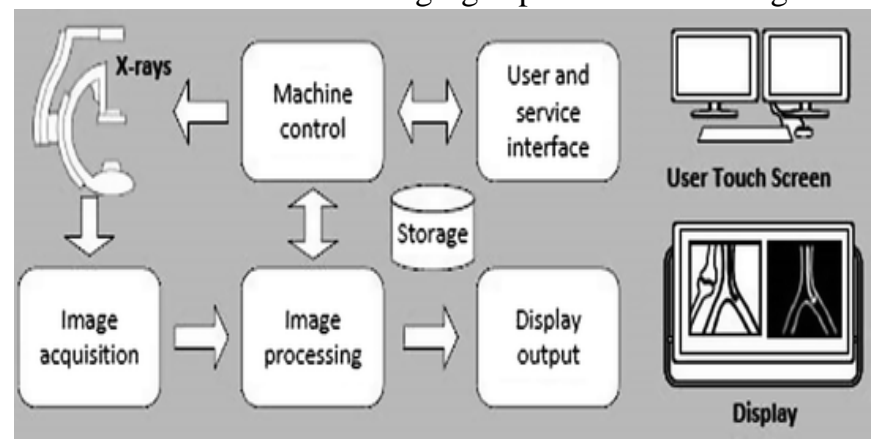

Figure 1: Image processing concept

\section{LITERATURE SURVEY}

Splits which occur due to any mishap is a bone fracture. Overall many kinds of bone fractures are there among them spiral, segmented, impacted, avulsed, oblige[1,2]. Mostly $\mathrm{X}$-Ray images are segmented by using methods like Canny edge detection, Sobel Edge Detection, Roberts[3-6].[4]It is mentioned that among various segmentation or a clustering technique Canny Edge detection technique performs better[7].K-Means clustering is another clustering technique that performs well in image segmentation[8]. Fuzzy C Means is a clustering technique that adds better clustering as it adds membership function [9]. Weighted $\mathrm{C}$ means clustering is also another kind of clustering technique that adds in better performance of the system [10]. Statistical feature are the best feature extraction techniques [10]. Classification of type of image aids an orthopedic. For classifying the X-Ray images, Local binary patterns along with the Random Forests are used[10]. For segmentation purpose kernel weighted C-Means clustering and also the automatic segmentation correctness is proposed [11]. Fuzzy based X-Ray classification is proposed [12]. For classifying $\mathrm{X}$-ray images texture feature extraction technique is proposed [13]. 


\section{PROPOSED METHODOLOGY}

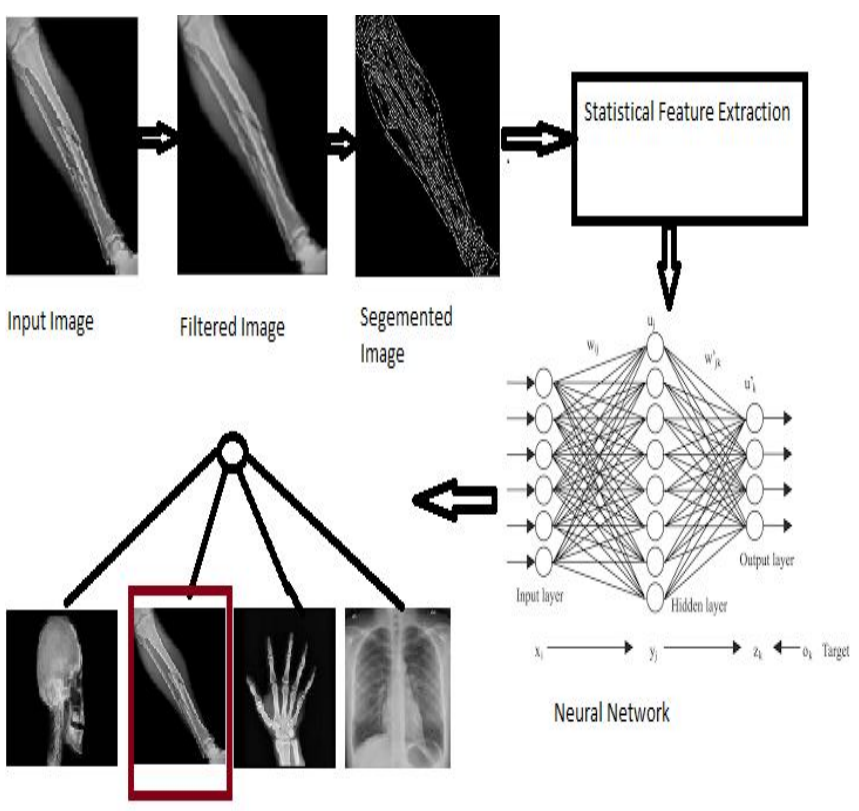

Figure 2: Architecture of proposed methodology

Figure 2 shows the architecture of proposed methodology where input image is given as input to the system and it is pre-processed first in order to remove the noise from the image. [14]Then the pre-processed image is fed as input for segmentation. Then from the segmented image statistical features are extracted which are given for training the classifiers[15].

\subsection{High Boost Filtering}

High boost filtering has a special characterization. It not only emphasized the high-frequency components but also it preserves the low-frequency components.[16] This makes to apply the high boost filtering for reprocessing. Here in the proposed work high boost filtering is applied for preprocessing as its gives more PSNR value.

$$
I_{h b}=I_{a p}+c I_{h p}=\left(W_{a p}+c W_{h p}\right) * I_{a}=W_{h b} * I_{a}
$$

Where $\mathrm{c}$ is a constant.

$$
W_{b b}=W_{a p}+c W_{b p}=\left[\begin{array}{lll}
0 & 0 & 0 \\
0 & 1 & 0 \\
0 & 0 & 0
\end{array}\right]+c\left[\begin{array}{ccc}
0 & -1 & 0 \\
-1 & 4 & -1 \\
0 & -1 & 0
\end{array}\right]=\left[\begin{array}{ccc}
0 & -c & 0 \\
-c & 4 c+1 & -c \\
0 & -c & 0
\end{array}\right]
$$

$$
W_{h}=W_{a q}+c W_{p q}=\left[\begin{array}{lll}
0 & 0 & 0 \\
0 & 1 & 0 \\
0 & 0 & 0
\end{array}\right]+c\left[\begin{array}{ccc}
-1 & -1 & -1 \\
-1 & 8 & -1 \\
-1 & -1 & -1
\end{array}\right]=\left[\begin{array}{ccc}
-c & -c & -c \\
-c & 8 c+1 & -c \\
-c & -c & -c
\end{array}\right]
$$

\subsection{Fuzzy c means}

Data can be made belongs to the 2 or more clusters using FCM(Fuzzy C Means).Membership functions are added to this and this makes FCM to work more for clustering when compared to other clustering techniques.

$$
\begin{gathered}
J_{m}=\sum_{i=1}^{H} \sum_{j=1}^{c} u_{i j}^{m}\left\|x_{i}-c_{j}\right\|^{2} \quad, \quad 1 \leq m<\infty \\
u_{i j}=\frac{1}{\sum_{k=1}^{c}\left(\frac{\left\|x_{i}-c_{j}\right\|}{\left\|x_{i}-c_{k}\right\|}\right)^{\frac{2}{m-1}}} \quad c_{j}=\frac{\sum_{i=1}^{H} u_{i j}^{m} \cdot x_{i}}{\sum_{i=1}^{N} u_{i j}^{m}} \\
\text { This iteration will stop when } \max _{i j}\left\{\left|u_{j i}^{(k+1)}-u_{i j}(k)\right|\right\}<\epsilon,
\end{gathered}
$$
where $\epsilon$ is for the termination criterion in between the values 0 and 1 ,whereas the $\mathrm{k}$ is used for looping. When there are 2 or more clusters are to be to be done fuzzy works better

\subsection{Statistical Feature Extraction}

For classification purpose some features are to be extracted here statistical features are taken .[17] Some of the statistical features here taken are kurtosis, entropy, and skewness, median.

\section{RESULTS AND DISCUSSION}

From a dataset of totally 2000 X-Ray images are collected from the NRI Medical Hospital, Department of Orthopaedics, Guntur, where 400 images are of skull out of which 200 are used for training and 200 are for testing,400 images are of Leg out of which 200 used for testing and 200 are used for training, 400 images are of hand out of which 200 are used for testing and 200 used for training,400 images are of chest out of which 200 are used for training and 200 are used for testing, and remaining 400 are neck out of which 200 are used for training and 200 are used for testing.Table.1 represents performance evaluation of $\mathrm{CNN}$ classifier and fig. 3 shows the statistical representation of CNN Classifier[18]. 
Table 1 :Performance evaluation of CNN

\begin{tabular}{|l|r|r|r|}
\hline Class & Accuracy & Specificity & Sensitivity \\
\hline Head & 94 & 90 & 89 \\
\hline Leg & 95 & 91 & 90 \\
\hline Hand & 93 & 88 & 87 \\
\hline Chest & 93 & 90 & 92 \\
\hline Neck & 94 & 89 & 90 \\
\hline
\end{tabular}

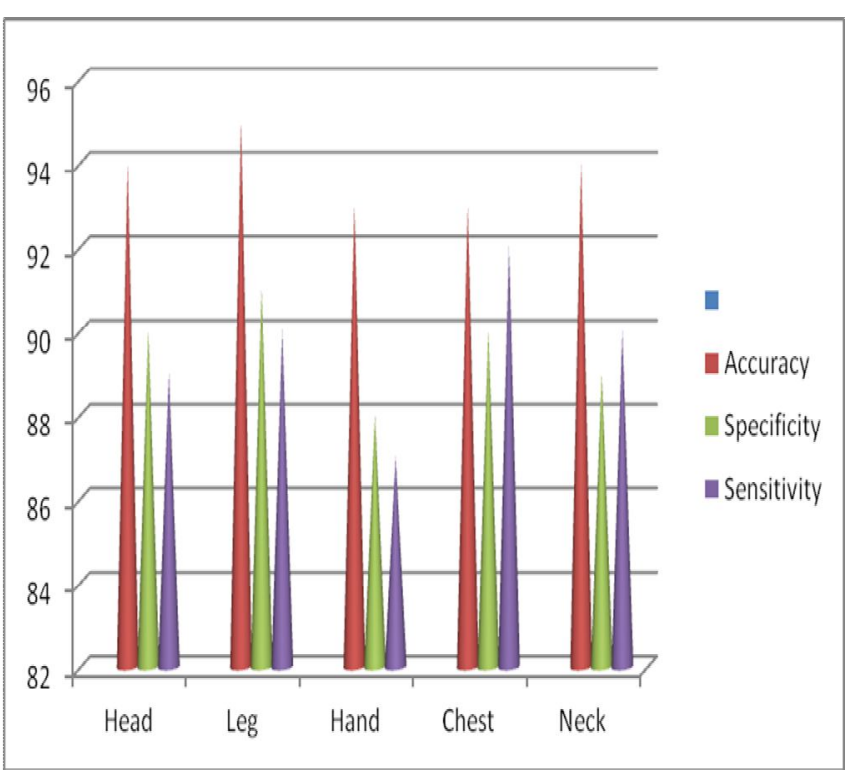

Figure 3 :Performance of classification using CNN

Table. 2 represents performance evaluation of SVM classifier and figure. 4 shows the statistical representation of SVM Classifier.

Table 2: Classification evaluation using SVM

\begin{tabular}{|l|r|r|r|}
\hline \multicolumn{4}{|c|}{ Classification evaluation of SVM } \\
\hline Class & Accuracy & Specificity & Sensitivity \\
\hline Head & 90 & 88 & 87 \\
\hline Leg & 89 & 89 & 88 \\
\hline Hand & 87 & 86 & 85 \\
\hline Chest & 89 & 88 & 90 \\
\hline Neck & 87 & 86 & 89 \\
\hline
\end{tabular}

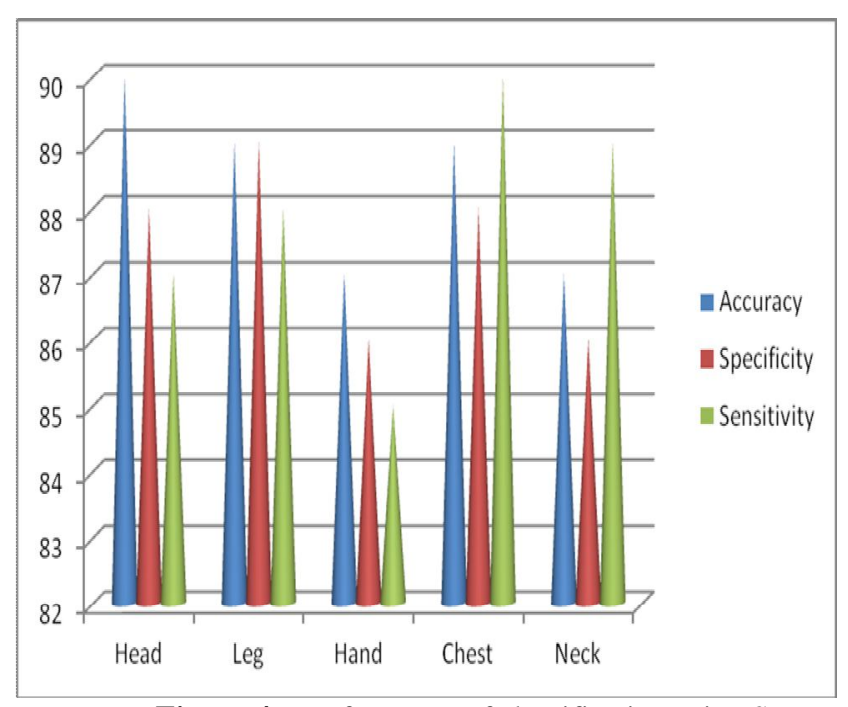

Figure 4 : Performance of classification using SVM

Table.3 represents performance evaluation of BPNN classifier and figure. 5 shows the statistical representation of BPNN Classifier.

Table 3: Classification evaluation using BPNN

\begin{tabular}{|l|r|r|r|}
\hline \multicolumn{4}{|c|}{ Classification evaluation of BPNN } \\
\hline Class & Accuracy & Specificity & Sensitivity \\
\hline Head & 88 & 87 & 86 \\
\hline Leg & 86 & 88 & 86 \\
\hline Hand & 85 & 84 & 83 \\
\hline Chest & 89 & 87 & 89 \\
\hline Neck & 85 & 86 & 89 \\
\hline
\end{tabular}

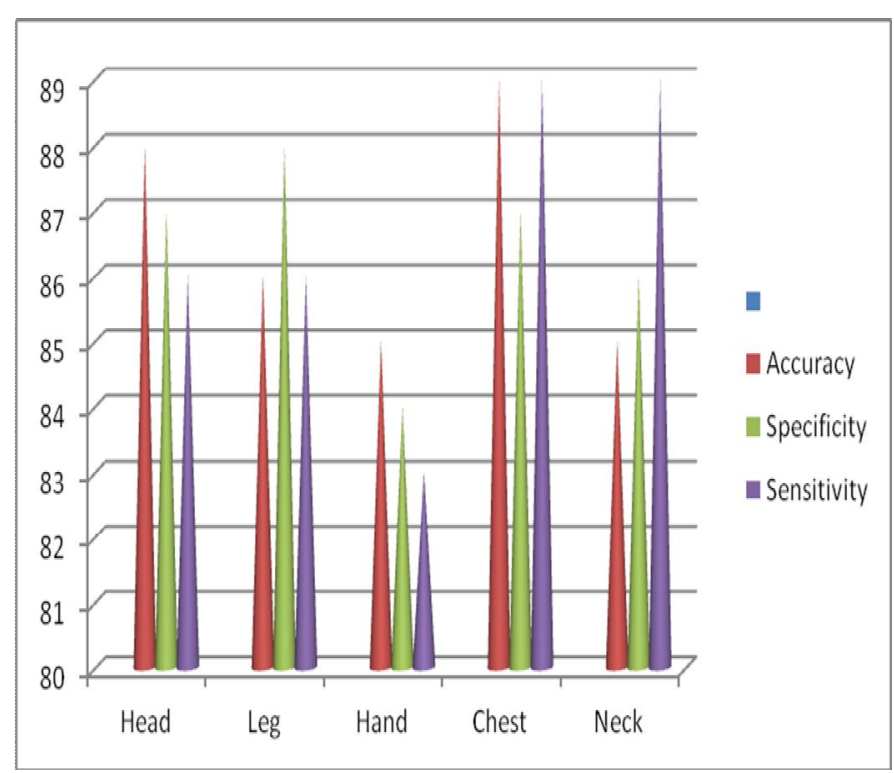

Figure 5: Performance of classification using BPNN 


\section{CONCLUSION}

Hence a proposed methodology works better compared to other classifiers.CNN proved to be best among the results in terms of accuracy when compared to other classifiers like SVM and BPNN. the accuracy rates with all classifiers where the Convolution neural network gives an accuracy rate of 94.2\% when compared to other neural networks like BPNN and SVM. Hence Convolution neural network $(\mathrm{CNN})$ is considered.

\section{FUTURE WORK}

This work can be extended to fracture detection of bone using $\mathrm{X}$-Ray images. Main purpose of classification of X-ray images is some works might be only for long bone Fracture detection and some might be only for curve bone fracture detection only. In this manner Classification of X-Ray images works as essential factor for fracture detection of bone using $\mathrm{X}$-Ray images.

\section{REFERENCES}

1. A.S.Bhide, Priyanka Patil, Shraddha Dhande,"Brain Segmentation using Fuzzy $C$ means clustering to detect tumor Region". International Journal of Advanced Research in Computer Science and Electronics Engineering Volume 1, Issue 2, April 2012.

2. Prateek Kumar Garg, Pushpneel Verma , Ankur Bhardwaz, "A Survey Paper on Various Median Filtering Techniques for Noise Removal from Digital Images" American International Journal of Research in Formal, Applied \& Natural Sciences, 7(1), June-August, 2014.

3. N.Abhirami, S.Karthik, M.Kanimozhi," Automated Brain Tumor Detection and Identification Using Medical Imaging". International Journal of Research in Computer Applications and Robotics ISSN 2320-7345.

4. Qingkun Song,Li Ma,JianKun Cao,Xiao Han," Image Denoising Based on Mean Filter and Wavelet Transform", 2015 4th International Conference on Advanced Information Technology and Sensor Application.

5. Arnlaug Wangensteen, Johannes L. Tol, Frank W. Roemer, Roald Bahr, "Intra- and interrater reliability of three different MRI grading and classification systems after acute hamstring injuries", 16th International Learning \& Technology Conference 2019.

6. Ruey-Maw Chen, Sheng-Chih Yang, Chuin-Mu Wang "MRI brain tissue classification using unsupervised optimized extenics-based methods", National Chin-Yi University of Technology, Taiping 411, Taiwan

7. Sanjeev Kumar, Chetna Dabas, Sunila Godara "Classification of Brain MRI Tumor Images: A
Hybrid Approach" Information Technology and Quantitative Management (ITQM2017)

8. Basha, C. Z., Sricharan, K. M., Dheeraj, C. K., \& Ramya Sri, R. (2018)."A study on wavelet transforms using image analysis". International Journal of Engineering and Technology (UAE), 7(2), 94-96.

9. C. Z. Basha, G. Rohini, A. V. Jayasri and S. Anuradha, "Enhanced and Effective Computerized Classification of X-Ray Images" 2020 International Conference on Electronics and Sustainable Communication Systems (ICESC), Coimbatore, India, 2020,pp.86-91,doi: 0.1109/ICESC48915.2020.9155788.

10. Basha, C. Z., Simha, G. K. J., \& Krishna, Y. V. (2019). "An efficient and robust fracture detection in femur bones". International Journal of Innovative Technology and Exploring Engineering, 9(1), 1954-1957.

11. C. Z. Basha, M. R. K. Reddy, K. H. S. Nikhil, P. S. M. Venkatesh and A. V. Asish, "Enhanced Computer Aided Bone Fracture Detection Employing X-Ray Images by Harris Corner Technique," 2020 Fourth International Conference on Computing Methodologies and Communication (ICCMC), Erode, India, 2020, pp. 991-995

12. Selvikvåg Lundervold, A., \& Lundervold, A. (2018). An overview of deep learning in medical imaging focusing on MRI. Zeitschrift Fur Medizinische Physik. doi:10.1016/j.zemedi.2018.11.002

13. Ibrahim, W. H., Osman, A. A. A., \& Mohamed, Y. I. (2013). MRI brain image classification using neural networks. 2013 INTERNATIONAL CONFERENCE ON COMPUTING, ELECTRICAL AND ELECTRONIC ENGINEERING (ICCEEE). doi:10.1109/icceee.2013.6633943

14. Khemphila, A., \& Boonjing, V. (2011). Heart Disease Classification Using Neural Network and Feature Selection. 201121 st International Conference on Systems Engineering. doi:10.1109/icseng.2011.80

15. C. Saha and M. F. Hossain, "MRI brain tumor images classification using K-means clustering, NSCT and SVM" 2017 4th IEEE Uttar Pradesh Section International Conference on Electrical, Computer and Electronics (UPCON), Mathura, 2017, pp. 329-333.

16. S. Chaplot, L.M. Patnaik, N.R. Jagannathan, "Classification of magnetic resonance brain images using wavelets as input to support vector machine and neural machine", Biomedical Signal Processing and Control, vol. 1, no. 1, pp. 86-92, January 2006.

17. Basha, C. Z., Krishna, A., \& Kumar, S. S. (2019). "Automatic article detection in a jumbled scene using point feature matching”. In Examining Fractal Image Processing and Analysis (pp. 188-195). IGI Global. https://doi.org/10.4018/978-1-7998-0066-8.ch009

18. Basha, C. Z., Krishna, A., \& Savarapu, P. R. (2019). "Automatic detection of lung infection". International Journal of Recent Technology and Engineering, 8(3), 200-203. 